\title{
Signatures of collective behavior in small systems
}

\author{
Kozlov I. ${ }^{\mathrm{a}}$, Luzum M. ${ }^{\mathrm{a}, \mathrm{b}}$, Denicol G. S. ${ }^{\mathrm{a}}$, Jeon S. ${ }^{\mathrm{a}}$, Gale C., \\ ${ }^{a}$ McGill University, Department of Physics, 3600 University Street, Montreal QC H3A 2T8, Canada \\ ${ }^{b}$ Lawrence Berkeley National Laboratory, Berkeley, CA 94720, USA \\ ${ }^{c}$ Frankfurt Institute for Advanced Studies, Ruth-Moufang-Str. 1, D-60438 Frankfurt am Main, Germany
}

\begin{abstract}
We perform 3+1D viscous hydrodynamics calculations of proton-nucleus (pA) and nucleus-nucleus (AA) collisions. Our goal is to understand the apparent collective behavior recently observed in pA collisions and to verify whether the highest multiplicity collision systems can be accurately described as a relativistic fluid. We compare our calculations of flow variables to existing measurements, and demonstrate that hydrodynamics correctly captures the measured trends. We show that our predictions for the pair correlation observable $r_{n}$ are validated by recent experimental pA measurements, and that our results are sensitive to the granularity of the initial state. We also compare our results with measurements done for nucleus-nucleus collisions.
\end{abstract}

Keywords: Heavy-ion collisions, quark-gluon plasma, hydrodynamics, flow, factorization

\section{Introduction}

It is currently believed that ultrarelativistic heavy-ion collisions at the Relativistic Heavy-Ion Collider (RHIC) and at the Large Hadron Collider (LHC) are able to reach temperatures high enough to create and study the quark-gluon plasma (QGP) in a controlled experimental environment. One of the most surprising results obtained at RHIC, and more recently, at the LHC, is that this novel state of nuclear matter behaves as an almost prefect fluid, with one of the smallest shear viscosity-to-entropy density ratios in nature.

The main point of colliding heavy ions instead of, e.g. protons, was always to create a system that is large enough to achieve or approach thermodynamic equilibrium. Only if this is accomplished it becomes possible to study the thermodynamic and transport properties of the bulk nuclear matter. Recently, some of the same signs of collective behavior initially observed in heavy-ion collisions more than a decade ago at RHIC, were also seen in high multiplicity p-Pb collisions measured at the LHC, by the ATLAS, CMS, and ALICE collaborations [1, 2, 3]. At RHIC, PHENIX observed similar signals in high multiplicity d-Au collisions [4], confirming the findings made at LHC energies. One should note that pA collisions were always considered as baseline and well understood measurements, and the fact that signs of hydrodynamic behavior were observed in such small systems came as a surprise. Understanding this novel behavior of strongly interacting QCD matter is now one of the main threads in high energy nuclear physics.

In this work we perform hydrodynamic simulations of pA and AA collisions at LHC energies. Our goal is to verify whether high multiplicity pA collisions are really able to create a quark-gluon plasma near thermodynamic equilibrium. We test our hydrodynamic model by comparing its results to the full set of the experimentally measured transverse momentum two-particle correlation matrix elements. We further check if pA and AA collisions can be described within the same hydrodynamic model.

Email address: ikozlov@physics.mcgill.ca (Kozlov I.) 


\section{Hydrodynamic model}

The initial state of the collision in our model is calculated using the Monte-Carlo Glauber model [5], extended by including the effects of system's longitudinal anisotropy and of local entropy density fluctuations $[6,7]$ as described below. The subsequent dynamics of the system is evolved using relativistic dissipative hydrodynamics, solved numerically in $3+1$ dimensions with the Music approach [8].

The initial entropy density profile at the thermalization time, $\tau_{0}=0.6 \mathrm{fm} / \mathrm{c}$, is given by

$$
s\left(\vec{x}_{\perp}, \eta, \tau_{0}\right)=\left(1 \pm \frac{\eta}{y_{\text {beam }}}\right) \exp \left[-\frac{\left(|\eta|-\eta_{0}\right)^{2}}{2 \sigma_{\eta}^{2}} \theta\left(|\eta|-\eta_{0}\right)\right] \times \sum_{i=1}^{N_{\text {part }}} \frac{S_{i}}{2 \pi \sigma^{2}} \exp \left(-\frac{\left|\vec{x}_{\perp}-\vec{x}_{\perp}^{i}\right|^{2}}{2 \sigma^{2}}\right),
$$

where $N_{\text {part }}$ is the number of wounded nucleons, $\left(\vec{x}_{\perp}^{i}, \eta^{i}\right)$ is the position of the $i$-th wounded nucleon in hyperbolic coordinates and \pm corresponds to the sign of the participating nucleon's longitudinal momentum. The wounded nucleons are calculated taking a nucleon-nucleon inelastic cross section of $\sigma_{\mathrm{NN}}=67 \mathrm{mb}$. The parameter $\sigma$ specifies the length scale of the entropy density fluctuations in the transverse plane and is taken to be in the range $\sigma=0.4-0.8$ $\mathrm{fm}$. Recalling multiplicity fluctuations occurring in proton-proton collisions, we use a negative binomial distribution (NBD) to describe individual participant's contribution, $S_{i}$, to the total entropy. To describe the entropy density profile in the longitudinal direction, we use parameters values $\eta_{0}=2.5, \sigma_{\eta}=1.4$ and $y_{\text {beam }}=8.58\left(\mathrm{p}-\mathrm{Pb}\right.$ at $\sqrt{s_{\mathrm{NN}}}=5.02$ $\mathrm{TeV})$.

In all our calculations the initial transverse velocity profile is assumed to be zero and the system starts its hydrodynamic evolution in local thermodynamic equilibrium. The hydrodynamic equations solved correspond to the usual continuity equation, $\partial_{\mu} T^{\mu \nu}=0$, which describes energy-momentum conservation, coupled with a version of Israel-Stewart theory [9],

$$
\tau_{\pi} \Delta_{\alpha \beta}^{\mu v} u^{\lambda} \partial_{\lambda} \pi^{\alpha \beta}+\pi^{\mu v}=2 \eta \sigma^{\mu \nu}+\frac{4}{3} \tau_{\pi} \pi^{\mu \nu} \partial_{\lambda} u^{\lambda}
$$

which describes the time evolution of the shear-stress tensor, $\pi^{\mu \nu}$. Above, we introduced the shear tensor, $\sigma^{\mu \nu}=$ $\Delta_{\alpha \beta}^{\mu \nu} \partial^{\alpha} u^{\beta}$, and the double, symmetric, traceless projection operator $\Delta_{\alpha \beta}^{\mu \nu}=\left(\Delta_{\alpha}^{\mu} \Delta_{\beta}^{v}+\Delta_{\beta}^{\mu} \Delta_{\alpha}^{v}\right) / 2-\Delta_{\alpha \beta} \Delta^{\mu \nu} / 3$. In this work, we neglect the effects of bulk viscous pressure and of net-baryon number diffusion. The equation of state employed in all our calculations is the parametrization of lattice QCD calculations by Huovinen and Petreczky [10]. Also, the shear viscosity coefficient is assumed to be proportional to the entropy density, $\eta / s=0 .-0.08$, and the shear relaxation time is given by $\tau_{\pi}=3 \eta /(\varepsilon+P)$, with $\varepsilon$ being the energy density and $P$ the thermodynamic pressure. The freezeout procedure is implemented via the Cooper-Frye formalism [11], with a freeze-out temperature of $T=150$ $\mathrm{MeV}$. We direct the reader to Ref. [12] for further details of our model and to Ref. [7], [13], [14] for the hydrodynamic simulations of pA collisions utilizing different types of initial conditions.

\section{Comparison with proton-nucleus data}

We start by comparing our calculations of the integrated two-particle and four-particle cumulants, $v_{n}\{2\}$ and $v_{n}\{4\}$, to the experimentally observed values. In Fig. 1 (a) we show integrated $v_{2}\{2\}$ and $v_{2}\{4\}$ as a function of multiplicity for pA collisions, with the curves corresponding to the theoretical predictions and the points to the CMS data [15]. We see that our calculations are in good agreement with the data, supporting the claim that high multiplicity pA collisions display collective behavior. Surprisingly, the agreement with the data remains good even when the multiplicity is not so large and only visibly starts to break when $N_{\text {offline }}<50$. We explored features of the hydro calculations, by modifying parameters of granularity $(\sigma)$ and viscosity $(\eta / s)$ in the value ranges specified above, and verifying, that hydro curves stay in the vicinity of the experimental data. Thus, we expect our hydro model to correctly capture the general experimental trends. To test it, we consider the CMS finding of the remarkably similar magnitude of the $v_{3}\{2\}$

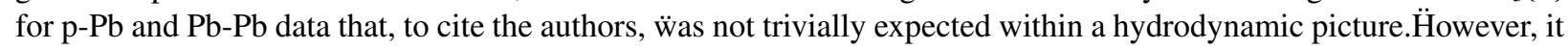
naturally follows in our hydro model without any additional fitting, Fig. 1 (b). We have not made efforts to reproduce the absolute magnitude of $v_{3}\{2\}$, but it appears plausible that the apparent collectivity observed in pA collisions can have a fluid-dynamical origin. 

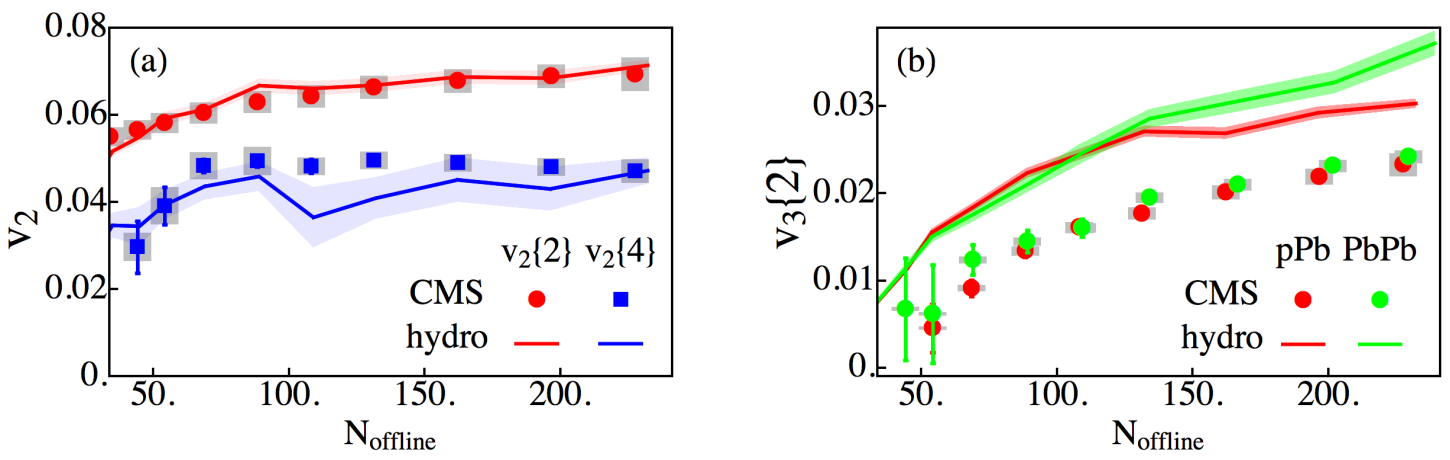

Figure 1. Elliptic flow variables compared to calculations from the hydrodynamic approach discussed in the text. The left panel shows CMS data for $\mathrm{p}-\mathrm{Pb}$ collisions at $\sqrt{s_{\mathrm{NN}}}=5.02 \mathrm{TeV}$. The right panel contains $\mathrm{p}-\mathrm{Pb}(5.02 \mathrm{TeV})$ and $\mathrm{Pb}-\mathrm{Pb}(2.76 \mathrm{TeV})$ triangular flow measurements with similar final multiplicities [15], along with results from calculations described in the text with the used parameters values $\sigma=0.4 \mathrm{fm}, \eta / s=0.08$.

A more stringent test of the fluid-dynamical nature of pA collisions can be obtained from a detailed analysis of the transverse momentum structure of two-particle correlations [16]. Experimentalists measure the full correlation matrix

$$
V_{n \Delta}\left(p_{T}^{a}, p_{T}^{b}\right)=\left\langle\frac{1}{N_{\text {pairs }}^{a, b}} \sum_{\text {pairs }\{a, b\}} \cos n \Delta \phi\right\rangle,
$$

- which includes the regularly discussed two-particle cumulant - with $N_{\text {pairs }}^{a, b}$ being the number of pairs with transverse momenta $p_{T}^{a}$ and $p_{T}^{b}$ in a given event, $\sum_{\text {pairs }\{a, b\}}$ is a summation over this set of pairs, and $\Delta \phi=\phi^{a}-\phi^{b}$ their relative azimuthal angle. The brackets denote an average over events. Note that the two-particle cumulant shown in Fig. 1 is a special case of this general correlator $v_{n}\{2\}=\sqrt{V_{n \Delta}\left(\bar{p}_{T}, \bar{p}_{T}\right)}$ with $\bar{p}_{T} \in[0.3,3.0] \mathrm{GeV} / \mathrm{c}$. While $v_{n}$ (Fig. 1) probes the overall magnitude of the correlation, considering comparison of the full matrix (and its elements' ratios) calculated in a hydro model to the experimentally measured one allows to better study the momentum structure of the correlations.

It is more convenient and intuitive to perform this comparison after changing variables from $V_{n \Delta}\left(p_{T}^{a}, p_{T}^{b}\right)$ to $r_{n}\left(p_{T}^{a}, p_{T}^{b}\right) \equiv V_{n \Delta}\left(p_{T}^{a}, p_{T}^{b}\right) / \sqrt{V_{n \Delta}\left(p_{T}^{a}, p_{T}^{a}\right) V_{n \Delta}\left(p_{T}^{b}, p_{T}^{b}\right)}$, because $r_{n}$ ratio is being bounded by \pm 1 in fluid-dynamical simulations [16]. As well, the fact that these bounds were satisfied [17] is non-trivial, since otherwise it would imply the failure of the hydrodynamic picture, regardless of the initial condition or set of parameters employed.
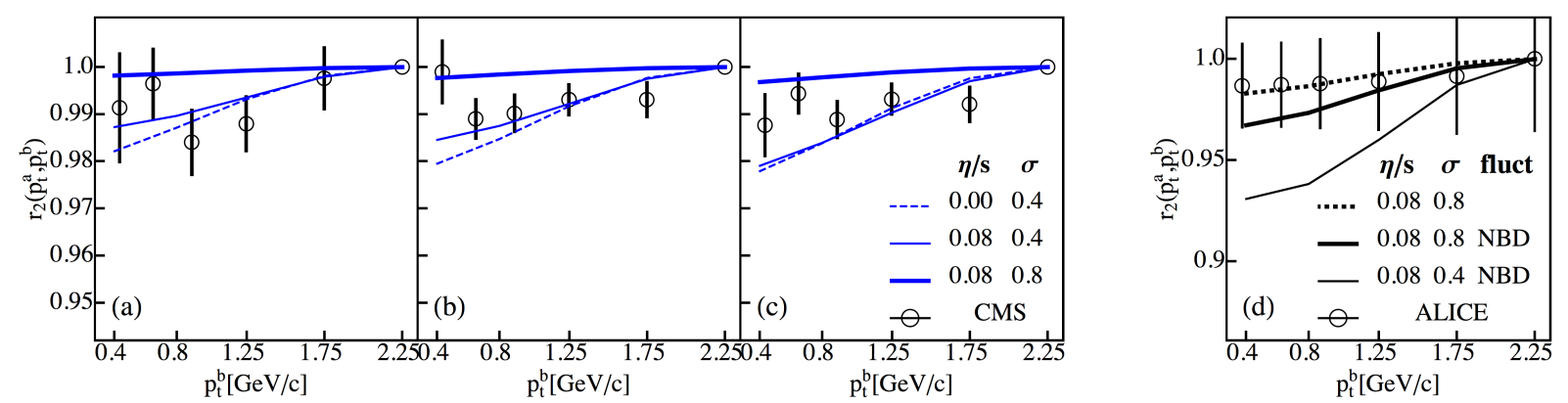

Figure 2. The pair correlation variable $r_{n}\left(p_{T}^{a}, p_{T}^{b}\right)$ (only $n=2$ case is shown), for a range of the trigger momentum $p_{T}^{a} \in[2,2.5] \mathrm{GeV} / \mathrm{c}$, as a function of the associate momentum $p_{T}^{b}$. Panels (a), (b), and (c): Panel (a) is for a multiplicity class of $220 \leq N_{\text {offline }}<260$, panel (b) is for $185 \leq N_{\text {offline }}<220$, and panel (c) is for $150 \leq N_{\text {offline }}<185$. The legend in panel (c) applies also to (a) and (b). The data is by CMS collaboration [17], and the curves are predictions [12] of the model described in the text (see also the model predictions for ALICE measurements [18]). Panel d: $r_{2}\left(p_{T}^{a}, p_{T}^{b}\right)$ for $\mathrm{Pb}-\mathrm{Pb}$ collisions as measured by the ALICE collaboration [19]. Details are given in the text.

In Fig. 2 (a-c), we show $r_{2}\left(p_{T}^{a}, p_{T}^{b}\right)$ for pA collisions as a function of $p_{T}^{b}$, for $p_{T}^{a} \in[2,2.5] \mathrm{GeV} / \mathrm{c}$ and for several multiplicity ranges. We have verified that our results imply a monotonic dependence of $r_{n}$ on the multiplicity, i.e., $r_{n}$ values decrease when we go to lower multiplicities. In order to probe the sensitivity of this observable to the 
granularity and viscosity of this system, we also show results in the ideal fluid limit, $\eta / s=0$, and with smaller granularity, $\sigma=0.8 \mathrm{fm}$. We observe that the effect of viscosity on $r_{n}$ is not very large, but that the effect of granularity is rather significant. The CMS data clearly favors the calculation with larger granularity, i.e., $\sigma=0.4 \mathrm{fm}$. The fact that $r_{n}$ exhibits this sensitivity to the granularity is beneficial, as it could be used as a probe of the initial condition's granularity in the same way flow is used to probe the viscous properties of the QCD matter.

In Fig. $2(\mathrm{~d})$, we show $r_{2}\left(p_{T}^{a}, p_{T}^{b}\right)$ for peripheral AA collisions as a function of $p_{T}^{b}$, for the same range in $p_{T}^{a}$ described above, compared to the ALICE data [19]. Here, we test the effect of granularity, by changing $\sigma=0.4$ fm to $\sigma=0.8 \mathrm{fm}$, and of multiplicity fluctuations, by removing the NBD fluctuation of the entropy produced by each wounded nucleon. While in AA collisions both these quantities have a very small effect on the usual flow observables, we see that they have a considerable effect on $r_{n}$. Surprisingly, the calculation with larger granularity and with multiplicity fluctuations, which could reasonably describe the CMS pA collision data, is not in good agreement with the ALICE AA data. It is puzzling that pA and AA data cannot be described with the same set of parameters, since one expects that the same type of fluid is created in both collisions. We will further investigate this in future work.

\section{Conclusions}

In this work we have shown that hydrodynamic models are able to reasonably describe a wide range of flow variables and studied behavior of the $r_{n}$ observable. This quantity provides a general test of the applicability of hydrodynamics (independent of the used parameters and the initial conditions) to the description of high multiplicity pA collisions. We have shown that $r_{n}\left(p_{T}^{a}, p_{T}^{b}\right)$ is sensitive to local entropy fluctuations and to the initial granularity of the fluid: it is thus able to probe aspects of the initial state of the collision that traditional flow observables cannot. Importantly, the fact that our prediction of the pair correlation observables $r_{2}$ and $r_{3}$ were subsequently confirmed by the CMS collaboration measurements adds credence to the line of reasoning presented here. Finally, our attempt to describe $r_{n}$ data for various HIC systems reveals that the simultaneous interpretation of the available AA and pA collision data within a unified hydrodynamical approach still currently appears challenging.

Acknowledgments: The authors thank P. Bozek for fruitful discussions. This work was supported in part by the Natural Sciences and Engineering Research Council of Canada, and in part by the the Director, Office of Energy Research, Office of High Energy and Nuclear Physics, Division of Nuclear Physics, of the U.S. Department of Energy under Contract No. DE-AC02-05CH11231. I. K. acknowledges support from the Canadian Institute of Nuclear Physics, G. S. D. acknowledges support through a Banting Fellowship of the Natural Sciences and Engineering Research Council of Canada.

\section{References}

[1] G. Aad et al. [ATLAS Collaboration], Phys. Rev. Lett. 110, 182302 (2013) [arXiv:1212.5198 [hep-ex]].

[2] S. Chatrchyan et al. [CMS Collaboration], Phys. Lett. B 718, 795 (2013) [arXiv:1210.5482 [nucl-ex]].

[3] B. Abelev et al. [ALICE Collaboration], Phys. Lett. B 719, 29 (2013) [arXiv:1212.2001 [nucl-ex]].

[4] A. Adare et al. [PHENIX Collaboration], Phys. Rev. Lett. 111, 212301 (2013) [arXiv:1303.1794 [nucl-ex]].

[5] M. L. Miller, K. Reygers, S. J. Sanders and P. Steinberg, Ann. Rev. Nucl. Part. Sci. 57, 205 (2007) [nucl-ex/0701025].

[6] P. Bozek and I. Wyskiel, Phys. Rev. C 81, 054902 (2010) [arXiv:1002.4999 [nucl-th]].

[7] P. Bozek, Phys. Rev. C 85, 014911 (2012) [arXiv:1112.0915 [hep-ph]].

[8] B. Schenke, S. Jeon and C. Gale, Phys. Rev. Lett. 106, 042301 (2011) [arXiv:1009.3244 [hep-ph]].

[9] W. Israel and J. M. Stewart, Annals Phys. 118, 341 (1979).

[10] P. Huovinen and P. Petreczky, Nucl. Phys. A 837, 26 (2010).

[11] F. Cooper and G. Frye, Phys. Rev. D 10, 186 (1974).

[12] I. Kozlov, M. Luzum, G. Denicol, S. Jeon and C. Gale, arXiv:1405.3976 [nucl-th].

[13] A. Bzdak, B. Schenke, P. Tribedy and R. Venugopalan, Phys. Rev. C 87, no. 6, 064906 (2013) [arXiv:1304.3403 [nucl-th]].

[14] K. Werner, M. Bleicher, B. Guiot, I. Karpenko and T. Pierog, Phys. Rev. Lett. 112, 232301 (2014) [arXiv:1307.4379 [nucl-th]].

[15] S. Chatrchyan et al. [CMS Collaboration], Phys. Lett. B 724, 213 (2013) [arXiv:1305.0609 [nucl-ex]]; Additional data on the CMS public wiki: https://twiki.cern.ch/twiki/bin/view/CMSPublic/PhysicsResultsHIN13002

[16] F. G. Gardim, F. Grassi, M. Luzum and J. -Y. Ollitrault, Phys. Rev. C 87, no. 3, 031901 (2013) [arXiv:1211.0989 [nucl-th]].

[17] CMS Collaboration [CMS Collaboration], CMS-PAS-HIN-14-012 ; D. Devetak et al.[CMS Collaboration], these proceedings.

[18] Y. Zhou [for the ALICE Collaboration], arXiv:1407.7677 [nucl-ex].

[19] K. Aamodt et al. [ALICE Collaboration], Phys. Lett. B 708, 249 (2012) [arXiv:1109.2501 [nucl-ex]]. 